\title{
A novel method for tizanidine hydrochloride determination in aqueous solution based on fluorescence quenching of functionalised CdS quantum dots as luminescent probes
}

\author{
Marwa Ahmed Fouad* and Ehab Farouk Elkady \\ Pharmaceutical Chemistry Department, Faculty of Pharmacy, Cairo University, Cairo-11562, Egypt \\ *Corresponding author at: Pharmaceutical Chemistry Department, Faculty of Pharmacy, Cairo University, Cairo-11562, Egypt. \\ Tel.: +2.012.2441198; fax: +2.022.4148452. E-mail address: merro911@yahoo.com (M.A. Fouad).
}

\section{ARTICLE INFORMATION}

\section{Received: 14 July 2011}

Received in revised form: 19 August 2011

Accepted: 21 August 2011

Online: 31 March 2012

\section{KEYWORDS}

Quantum dots

Determination

Nanotechnology

Fluorescence quenching

Tizanidine hydrochloride

Pharmaceutical preparation

\section{ABSTRACT}

A novel, sensitive and convenient method for the determination of tizanidine hydrochloride (TZD) based on the fluorescence quenching of thioglycolic acid-capped CdS quantum dots (TGA-CdS QDs) is proposed. Luminescent CdS semiconductor quantum dots modified by thioglycolic acid were synthesized from cadmium nitrate and sodium sulfide in alkaline aqueous solution. The modified CdS QDs are water-soluble, stable and highly luminescent. When TZD was added into the CdS QDs colloidal solution, the surface of CdS QDs generates the electrostatic interaction in aqueous medium, which induces the quenching of fluorescence emission at $518 \mathrm{~nm}$ upon excitation at $340 \mathrm{~nm}$. Under the optimal conditions, the SternVolmer calibration plot of $\mathrm{F}_{\mathrm{o}} / \mathrm{F}$ against concentration of TZD was linear in the range of 3.0$18.0 \mu \mathrm{g} / \mathrm{mL}$ with a correlation coefficient of 0.9953 . The detection limit was $1.35 \mu \mathrm{g} / \mathrm{mL}$. The relative standard deviation for five determinations of $9 \mu \mathrm{g} / \mathrm{mL}$ TZD was $2.29 \%$. The proposed method was successfully applied to commercial tablets with satisfactory results. The results were found to be in good agreement with those obtained by the reference method. The possible fluorescence quenching mechanism for the reaction is also discussed.

\section{Introduction}

Tizanidine (TZD), 5-chloro- $N$-(4,5-dihydro- $1 H$-imidazol-2yl)benzo[c][1,2,5]thiadiazol-4-amine is an $\alpha_{2}$-adrenergic agonist and centrally active myotonolytic skeletal muscle relaxant with a chemical structure unrelated to other muscle relaxants [1]. It reduces spasticity by increasing presynaptic inhibition of motor neurons. It also reduces increased muscle tone associated with spasticity in patients with multiple sclerosis or spinal cord injury. Two and four mg tablets are available for oral administration.

Few methods have been described for the determination of TZD in pharmaceutical preparations or biological fluids. These methods include spectrophotometry [2,3], gas chromatography-mass spectrometry [4], HPTLC [1,5] and HPLC [1,6-8]. Besides, a radioimmunoassay method for the quantification of TZD hydrochloride has been described [9]. It is worth noting that no previous spectrofluorometric method has been reported for the determination of TZD.

The fluorescence probe method has been increasingly employed due to its simplicity, selectivity, and cost-effective nature $[10,11]$. As a kind of novel fluorescence probe, quantum dots (QDs) have always been used in biology and medicine recently, especially in analytical chemistry [12].

Compared to organic fluorophores, QDs exhibit higher quantum yield, color availability, good photo-stability, large surface-to-volume ratio and surface functionality [10]. Thus, QDs possess highly attractive fluorescent properties to be ideal fluorescent indicators for chemical and biological assay.

Direct aqueous-phase synthesis of semiconductor nanocrystals with high photoluminescence quantum yield would provide a convenient, economic, and environmentally friendly alternative to the labor-intensive method of organic-phase synthesis followed by ligand exchange. CdS nanocrystals represent one material that can be both fabricated and functionalized in a single synthetic step [13]. Compatible with aqueous assay condition, the surface modified quantum dots are usually capped with some appropriate hydrophilic functional reagents used as stabilizing agents, such as L-cysteine, thioglycollic acid (TGA) and so on. These surface modification reagents of QDs may change their optical and chemical properties. On one hand, these agents can modify the surface of QDs to diminish the QDs' crystal defect. So the intensity of fluorescence would be much stronger. On the other hand, QDs would be hydrophilic, so they could participate in the reaction in aquatic condition. The interactions between some substances and the modified quantum dots would change their photophysical and photochemical properties [14].

Due to the lack of spectrofluorometric methods for the determination of TZD, the aim of the present work was to develop a sensitive, reproducible and simple spectrofluorometric method for TZD determination in bulk or in tablets. TZD has no native fluorescence, thus, it was deemed useful to investigate the interaction between thioglycolic acid-capped CdS quantum dots (TGA-CdS QDs) and TZD. As a result, it was found that the fluorescence intensity of CdS QDs is quenched in the presence of TZD in aqueous medium. The quenched intensity of fluorescence was proportional to the concentration of TZD. Based on this phenomenon, a new method for the determination of TZD by this fluorescent technique was developed. The method has been applied in the determination of TZD in commercial tablets with satisfactory results. 


\section{Experimental}

\subsection{Instrumentation}

The spectra and intensity of fluorescence were measured with a RF-1501 Shimadzu spectrofluorophotometer (Japan). UV-vis absorption spectra were recorded with a Shimadzu Ultraviolet/visible recording spectrophotometer UV-1600 (Japan). The transmission electron microscopic (TEM) images of the nanoparticles were obtained using a JEM-2100 transmission electron microscope (JEOL, Japan).

\subsection{Reagents and reference samples}

Pharmaceutical grade of TZD and Sirdalud ${ }^{\circledR}$ tablets (batch number Y0045) nominally containing TZD (4 mg), were kindly supplied by Novartis Pharma Egypt (Cairo, Egypt). Potassium dihydrogen phosphate and orthophosphric acid (85\%) were purchased from VWR Chemicals (Pool, England). Bi-distilled water was produced in-house (Aquatron Water Still, A4000D, UK). Cadmium nitrate, sodium sulphide and thioglycolic acid (extra-pure, 80\%) were purchased from Loba Chemie PVT LTD (India). All other chemicals and reagents used were of analytical grade unless indicated otherwise. Standard stock solution of TZD $(0.1 \mathrm{mg} / \mathrm{mL})$ was prepared by dissolving $10 \mathrm{mg}$ of the drug in $100 \mathrm{~mL}$ water in a volumetric flask and then the required concentrations were prepared by serial dilutions.

\subsection{Procedure}

\subsubsection{Preparation of TGA-capped CdS QDS}

Functionalized TGA capped CdS QDs were synthesized via the procedure described by Winter et al. [13], with some modifications. $0.3 \mathrm{~mL}$ TGA were added to $10 \mathrm{~mL}$ of $15 \mathrm{mM}$ $\mathrm{Cd}\left(\mathrm{NO}_{3}\right)_{2}$. The mixture was adjusted to $\mathrm{pH}=7.0 \pm 0.05$ by dropwise addition of $0.5 \mathrm{M} \mathrm{NaOH}$. Ten milliliters of $6 \mathrm{mM}$ $\mathrm{Na}_{2} \mathrm{~S}_{9} 9 \mathrm{H}_{2} \mathrm{O}$ solution were added to this solution with rapid stirring, which raised the $\mathrm{pH}$ to $\sim 8.5$. The reaction mixture was stirred for $2 \mathrm{~h}$. The solution was quantitavely transferred into $100 \mathrm{~mL}$ volumetric flask and completed to volume with water. The CdS QDs made according to this procedure are about $4 \mathrm{~nm}$.

\subsubsection{Standard procedure for spectrofluorometric determination of TZD}

In a $10 \mathrm{~mL}$ volumetric flask, $0.3 \mathrm{~mL}$ of CdS QDs solution and an appropriate volume of TZD standard stock solution or sample solution were transferred. After 10 minutes, the mixture was diluted to volume with water and mixed thoroughly. The fluorescence intensity of the solution was recorded at $518 \mathrm{~nm}$ with the excitation wavelength of $340 \mathrm{~nm}$. Both slit widths of excitation and emission were $10 \mathrm{~nm}$.

\subsubsection{Sample treatment}

For the pharmaceutical analysis, 10 tablets were weighed and powdered in a mortar. An accurately weighed amount of the powder equivalent to $10 \mathrm{mg}$ TZD was extracted with water $(3 \times 20 \mathrm{~mL})$ and filtered into a $100 \mathrm{~mL}$ volumetric flask. The solution was completed to volume with water to obtain a concentration equivalent to $0.1 \mathrm{mg} / \mathrm{mL}$ TZD.

\section{Results and discussion}

\subsection{Characterization of TGA capped CdS QDS}

The water soluble, TGA functionalized, CdS QDs were successfully synthesized according to the protocol described in section 2. The average size of the particle is about $4 \mathrm{~nm}$ as analyzed by TEM (Figure 1). The TEM image shows clearly that these QDs are monodisperse. The optical properties of TGA capped CdS QDs were characterized by UV-visible absorption spectrometry and fluorescence spectroscopy, which are powerful tools to confirm quantum-confined property of semiconductor QDs, as shown in Figure 2. The emission and excitation wavelengths of the particles were $518 \mathrm{~nm}$ and 340 $\mathrm{nm}$, respectively.

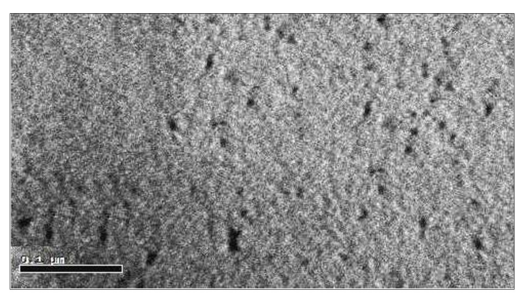

Figure 1. Transmission electron microscopy image of water-soluble TGA-CdS quantum dots.

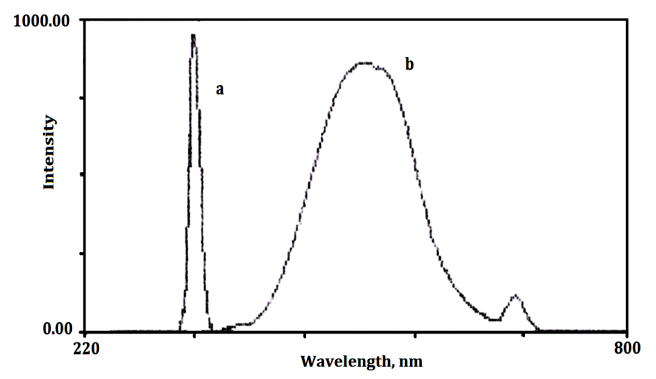

Figure 2. Excitation at $340 \mathrm{~nm}$ (a) and emission at $518 \mathrm{~nm}$ (b) spectra of TGA-CdS quantum dots.

\subsection{Reaction time and stability}

Fluorescence spectra were recorded at different time intervals after the addition of TZD into the QDs solution, Figure 3. The resulted spectra showed that all reactions were completed within $5 \mathrm{~min}$, hence, the fluorescence spectrum was recorded at $10 \mathrm{~min}$ after the addition of TZD. The fluorescence signals of the adduct were stable for more than $180 \mathrm{~min}$. Therefore, this system exhibits rapid reaction and good stability. It is worth noting that the prepared TGA capped CdS QDs were found to be stable for at least two months.

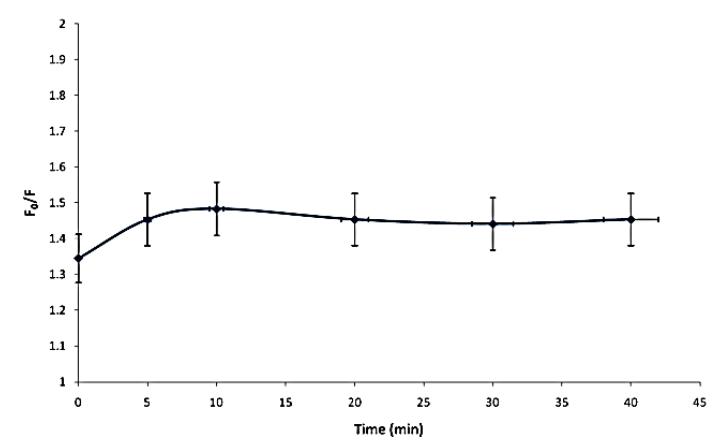

Figure 3. Effect of reaction time $1 \mathrm{~mL}$ TGA-CdS QDs; $9 \mu \mathrm{g} / \mathrm{mL}$ tizanidine.

\subsection{Effect of $\mathrm{pH}$}

The effect of $\mathrm{pH}$ in a range between 3.0 and 6.0 was studied in order to select the optimum conditions for the determination. Phosphate buffer solution $(20 \mathrm{mM})$ was used to adjust the acidity of the aqueous medium. The results showed that $\mathrm{pH}$ influences the fluorescence intensity of both QDs and QDs after adding TZD. The maximum value of relative 
fluorescence intensity $\left(\mathrm{F}_{\mathrm{o}} / \mathrm{F}\right)$ was obtained in the $\mathrm{pH}$ of 5.0. However, optimum linearity with the highest correlation coefficient was obtained when the study was conducted without the addition of phosphate buffer.

\subsection{Mechanism of the interaction of TGA-CdS QDs with tizanidine}

Quenching of FL emission from QDs may occur by several mechanisms: energy transferring, charge diverting, surface absorption, and surface bound complexation equilibrium attraction [10]. To explore the reason of fluorescence intensity decrease, the UV-vis absorption spectra of TGA-CdS QDs were investigated in the absence and presence of TZD, Figure 4. The results showed that TZD had no absorption in the $330-460 \mathrm{~nm}$ wavelength range. Thus, we can get that the quenching effect of the fluorescence of TGA-CdS QDs added TZD was not attributed to the absorption of the emission wavelength by TZD. From Figure 5, we can observe a blue-shift with increase in concentration of TZD on the emission spectra maximum. Thus, we believe that the TZD binding to the TGA-CdS QDs made the surface of the particles change, which induce the quench of QDs fluorescence. Besides, no major change was observed for the TGA-CdS QDs absorption spectra before and after adding TZD, which also means TGA-CdS QDs do not aggregate or become smaller after adding TZD [15].

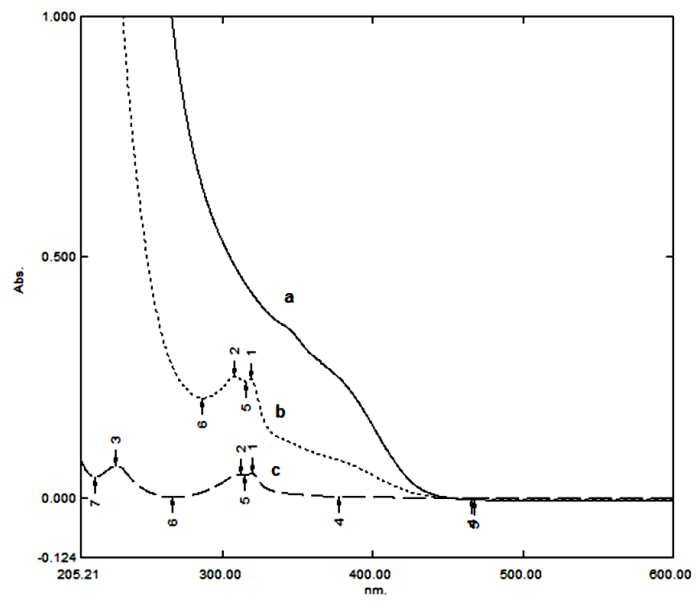

Figure 4. Absorption spectra of TGA-CdS QDs (a), CdS QDs adding tizanidine (b) and tizanidine (c).

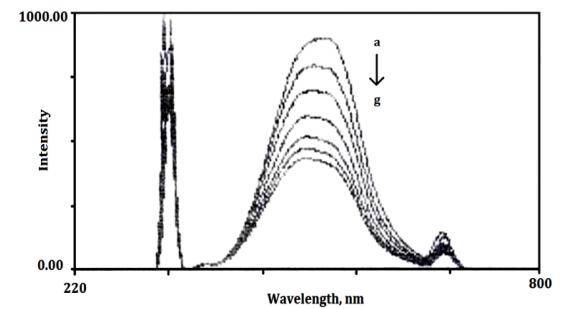

Figure 5. Effect of tizanidine on the emission of TGA-CdS QDs. The concentrations of tizanidine were (a) $0 \mu \mathrm{g} / \mathrm{mL}$, (b) $3 \mu \mathrm{g} / \mathrm{mL}$, (c) $6 \mu \mathrm{g} / \mathrm{mL}$, (d) $9 \mu \mathrm{g} / \mathrm{mL}$, (e) $12 \mu \mathrm{g} / \mathrm{mL}$, (f) $15 \mu \mathrm{g} / \mathrm{mL}$, and (g) $18 \mu \mathrm{g} / \mathrm{mL}$.

\subsection{Calibration, precision and accuracy}

The fluorescence spectra of TGA-CdS QDs with TZD were recorded at optimum experimental conditions. The fluorescence intensity of TGA-CdS QDs was significantly decreased with the addition of TZD (Figure 5). Considering this significant quenching of fluorescence intensity, the possibility of developing this into a sensitive method for TZD was evaluated. It was found that TZD quenched the fluorescence of QDs in concentration-dependent manner that were best described by the Stern-Volmer relationship, which is given by Equation 1.

$\mathrm{F}_{0} / \mathrm{F}=1+\mathrm{Ksv}[\mathrm{C}]$

$\mathrm{F}_{0}$ and $\mathrm{F}$ are the fluorescence intensity of the TGA-CdS QDs at $\lambda_{\text {em }}$ when excited at $\lambda_{\text {ex }}$ in the absence and presence of TZD, respectively. $[\mathrm{C}]$ is the concentration of TZD, and $\mathrm{K}_{\mathrm{Sv}}$ is the Stern-Volmer quenching constant, which was found to be 0.0582. Under optimal conditions, the relative fluorescence intensities of TGA-CdS QDs were decreased linearly with increasing TZD in the range of 3.0 to $18.0 \mu \mathrm{g} / \mathrm{mL}$ (Figure 6). The linear regression equation is $\mathrm{F}_{0} / \mathrm{F}=0.0635 \mathrm{x} \mathrm{C}+0.9724$. The correlation coefficient $\left(\mathrm{r}^{2}\right)$ is 0.9953 . The limit of detection $(\mathrm{S} / \mathrm{N}=3)$ was $1.35 \mu \mathrm{g} / \mathrm{mL}$.

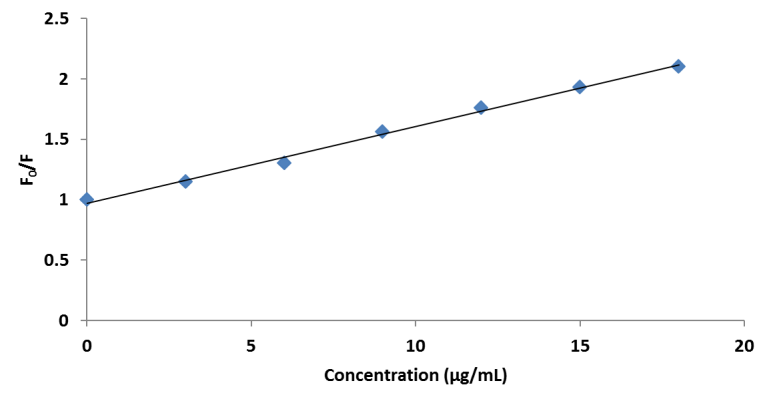

Figure 6. Stern-Volmer plot of tizanidine concentration dependence of the FL intensity of QDs with a 0.9958 correlation coefficient.

To assess the precision of the method, determinations were carried out for a set of 5 measurements of $9 \mu \mathrm{g} / \mathrm{mL}$ TZD under optimal conditions. The \%R.S.D. for five determinations was $2.29 \%$.

Accuracy of the results was calculated by \% recovery of 6 different concentrations of TZD other than those of the calibration curve and done by another analyst and on another instrument of the same type to test the reproducibility of the method as well. The results obtained including the mean of the recovery and standard deviation are displayed in Table 1.

Table 1. Statistical comparison between the results of the proposed method and the reference method for the determination of tizanidine hydrochloride

\begin{tabular}{lll}
$\begin{array}{l}\text { Statistical } \\
\text { Term }\end{array}$ & $\begin{array}{l}\text { Reference } \\
\text { Methoda,b }\end{array}$ & $\begin{array}{l}\text { Spectrofluorometric } \\
\text { Method }\end{array}$ \\
\hline Mean & 100.12 & 100.47 \\
S.D. \pm & 1.34 & 1.33 \\
S.E. \pm & 0.55 & 0.54 \\
$\%$ R.S.D. & 1.34 & 1.32 \\
$\mathrm{~N}$ & 6 & 6 \\
$\mathrm{~V}$ & 1.80 & 1.77 \\
$\mathrm{t}\left({ }^{*} 2.306\right)$ & 0.45 & \\
$\mathrm{~F}\left({ }^{*} 6.39\right)$ & 1.02 & \\
\hline a Figures in parentheses are the theoretical t and F values at $(p=0.05)$.
\end{tabular}

a Figures in parentheses are the theoretical $\mathrm{t}$ and $\mathrm{F}$ values at $(p=0.05)$.
b Company's method (Spectrophotometric method at $\lambda_{\max } 320 \mathrm{~nm}$ in water).

\subsection{Tolerance of foreign substance}

The tablets often contained the following excipients: microcrystalline cellulose, lactose, silicon dioxide colloidal and stearic acid; while in capsules, the main diluent is sucrose. In order to assess the selectivity of the proposed method, the tolerance of levels of coexisting excipients was tested by preparing solutions of TZD and different concentration of each excipient. The solution was mixed thoroughly and was added to TGA-CdS QDs solution. Most of the excipients are tolerated at relatively low levels $( \pm 3 \%)$. Accordingly, the excipients have hardly any effect on fluorescence of CdS QDs and the method has a high selectivity. So the method is practical and valuable. 


\subsection{Application}

The present method was applied to determine TZD in Sirdalud ${ }^{\circledR}$ tablets. Results are given in Table 2. The \%R.S.D. was $2.12 \%$, and the results obtained by the present method agreed with the labeled values for TZD tablets.

\begin{tabular}{llll}
\multicolumn{4}{l}{ Table 2. Analysis of Sirdalud ${ }^{\circledR}$ by the proposed procedure. } \\
$\begin{array}{llll}\text { Labeled amount } \\
(\mu \mathrm{g} / \mathrm{mL})\end{array}$ & $\begin{array}{l}\text { Proposed procedure } \\
(\boldsymbol{\mu g} / \mathrm{mL})\end{array}$ & $\begin{array}{l}\text { RSD } \\
(\%)\end{array}$ & $\begin{array}{l}\text { R\% } \\
(\mathbf{n}=6)\end{array}$ \\
\hline 9.00 & 8.80 & 2.12 & $96.51-100.11$ \\
\hline
\end{tabular}

\subsection{Statistical analysis}

Statistical analysis of the results obtained by the proposed method and the reference method for TZD was carried out by "SPSS statistical package version 11". The significant difference between the reference method and the described method was tested by one way ANOVA (F-test) at $p=0.05$ as shown in Table 1. The test ascertained that there was no significant difference between the two methods.

\section{Conclusion}

The proposed method for the determination of TZD is the first spectrofluorometric method reported for the drug and has the advantages of simplicity, precision, accuracy and convenience. In this method, TZD analysis has been developed based on the quenching of the fluorescence of thioglycolic acidcapped CdS quantum dots. When the proposed method was applied in the determination of TZD in tablets, the results were in good agreement with the labeled amount showing no interference from excipients. The proposed method was validated showing satisfactory data for all method validation parameters tested. The developed method can be conveniently used by quality control laboratories.

\section{References}

[1]. Kaul, N.; Dhaneshwar, S. R.; Agrawal, H.; Kakad, A.; Patil, B. J. Pharm. Biomed. Anal. 2005, 37, 27-38.

[2]. Sujatha, K.; Chitra, K.; Hettiarachchi, D. S.; Krishna, V. M.; Vasantha, J. Indian J. Pharm. Sci. 2003, 65, 519-520.

[3]. Shankar, M. B.; Shah, D. A.; Geetha, M.; Mehta, F. A.; Mehta, R. S.; Bhatt, K. K. Indian J. Pharm. Sci. 2004, 66, 332-336.

[4]. Lee, J.; Seo, J. H.; Kim, D. H. Analyst 2002, 127, 917-920.

[5]. Mahadik, K. R.; Paradkar, A. R.; Agrawal, H.; Kaul, N. J. Pharm. Biomed. Anal. 2003, 33, 545-552.

[6]. Qi, M.; Wang, P.; Wang, L. Anal. Chim. Acta. 2003, 478, 171-177.

[7]. Gandhimathi, M.; Ravi, T. K.; Varghese, S. J. J. Pharm. Biomed. Anal. 2005, 37, 183-185.

[8]. Battu, P. R. Int. J. ChemTech Res. 2009, 1, 499-501.

[9]. Healzlewood, V.; Symoniw, P.; Maruff, P.; Eadie, M. J. Eur. J. Clin. Pharmacol. 1983, 25, 65-72.

[10]. Peng, J.; Hu, X. J. Lumin. 2011, 131, 952-955.

[11]. Gore, A. H.; Mote, U. S.; Tele, S. S.; Anbhule, P. V.; Rath, M. C.; Patil, S. R.; Kolekar, G. B. Analyst 2011, 136, 2606-2612.

[12]. Dong, F.; Hu, K.; Han, H.; Liang. J. Microchim. Acta 2009, 165, 195-201.

[13]. Winter, J. O.; Gomez, N.; Gatzert, S.; Schmidt, C. E.; Korgel, B. A. Colloids Surf. A: Physicochem. Eng. Aspects. 2005, 254, 147-157.

[14]. Liu, M.; Xu, L.; Cheng, W.; Zeng, Y.; Yan, Z. Spectrochim. Acta Part A 2008, 70, 1198-1202.

[15]. Liang, J. G.; Huang, S.; Zeng, D. Y.; He, Z. K.; Ji, X. H.; Ai, X. P.; Yang, X. H. Talanta 2006, 69, 126-130. 